\title{
Instantaneous Midbrain Control of Saccade Velocity
}

\author{
Ivan Smalianchuk, ${ }^{1,3}$ @Uday K. Jagadisan, ${ }^{1,3}$ and $@$ Neeraj J. Gandhi $\mathbf{i}^{1,2,3}$ \\ ${ }^{1}$ Department of Bioengineering, ${ }^{2}$ Department of Neuroscience, and ${ }^{3}$ Center for Neural Basis of Cognition, University of Pittsburgh, Pittsburgh, \\ Pennsylvania 15213
}

The ability to interact with our environment requires the brain to transform spatially represented sensory signals into temporally encoded motor commands for appropriate control of the relevant effectors. For visually guided eye movements, or saccades, the superior colliculus (SC) is assumed to be the final stage of spatial representation, and instantaneous control of the movement is achieved through a rate code representation in the lower brain stem. We investigated whether SC activity in nonhuman primates (Macaca mulatta, 2 male and 1 female) also uses a dynamic rate code, in addition to the spatial representation. Noting that the kinematics of amplitude-matched movements exhibit trial-to-trial variability, we regressed instantaneous SC activity with instantaneous eye velocity and found a robust correlation throughout saccade duration. Peak correlation was tightly linked to time of peak velocity, the optimal efferent delay between SC activity and eye velocity was constant at $\sim 12 \mathrm{~ms}$ both at onset and during the saccade, and SC neurons with higher firing rates exhibited stronger correlations. Moreover, the strong correlative relationship and constant efferent delay observation were preserved when eye movement profiles were substantially altered by a blink-induced perturbation. These results indicate that the rate code of individual SC neurons can control instantaneous eye velocity and argue against a serial process of spatial-to-temporal transformation. They also motivated us to consider a new framework of saccade control that does not incorporate traditionally accepted elements, such as the comparator and resettable integrator, whose neural correlates have remained elusive.

Key words: motor execution; movement variability; efference copy; local feedback model; neural integrator; oculomotor

\section{Significance Statement}

All movements exhibit time-varying features that are under instantaneous control of the innervating neural command. At what stage in the brain is dynamical control present? It is well known that, in the skeletomotor system, neurons in the motor cortex use dynamical control. In the oculomotor system, in contrast, instantaneous velocity control of saccadic eye movements is not thought to be enforced until the lower brainstem. Using correlations between residual signals across trials, we show that instantaneous control of saccade velocity is present earlier in the visuo-oculomotor neuraxis, at the level of superior colliculus. The results require us to consider alternate frameworks of the neural control of saccades.

\section{Introduction}

Even when we want to produce the same movement repeatedly, our action exhibits heterogeneity across repetitions. Thus, variability in intended identical swings of a golf club, for example, yield different trajectories of the club, and therefore the ball. It is possible that variability in the swing could be the result of biological noise in the effectors, although a more likely explanation points to a neural origin (Carmena et al., 2005; Churchland et al.,

\footnotetext{
Received April 16, 2018; revised Sept. 18, 2018; accepted Sept. 22, 2018.

Author contributions: I.S., U.K.J., and N.J.G. edited the paper; U.K.J. and N.J.G. designed research; U.K.J. performed research; I.S. and U.K.J. analyzed data; I.S., U.K.J., and N.J.G. wrote the paper.

This work was supported by National Institute of Health Grants R01 EY022854, R01 EY024831, F31 EY027688, T32 DC011499, and P30 EY008098. We thank the two anonymous reviewers for astute insights.

The authors declare no competing financial interests.

Correspondence should be addressed to Dr. Neeraj J. Gandhi, University of Pittsburgh, 203 Lothrop Street, Pittsburgh, PA 15213. E-mail: neg8@pitt.edu.

https://doi.org/10.1523/JNEUROSCI.0962-18.2018

Copyright $\odot 2018$ the authors $\quad 0270-6474 / 18 / 3810156-12 \$ 15.00 / 0$
}

2006a; van Beers, 2007, 2008). While potential neural sources of movement variability have been extensively studied for hand movements (for review, see Churchland, 2015), less is known for eye movements, particularly the ballistic type known as saccades.

The superior colliculus (SC), a laminar subcortical structure with a topographic organization of the saccade motor map, is a central node in the oculomotor neuraxis (Gandhi and Katnani, 2011; Basso and May, 2017). It is intimately linked to the spatiotemporal transformation, in which visuo-oculomotor signals in the SC conform to a space or place code, whereas recipient structures in the brainstem exhibit a rate code. In a slight modification to this framework, the so-called dual-coding hypothesis (Sparks and Mays, 1990; Waitzman et al., 1991), saccade amplitude and direction are computed from the locus of population activity in the SC, whereas movement velocity is a "determinant" of the firing rate. The strongest evidence for SC control of saccade velocity comes from causal studies demonstrating that peak eye velocity is correlated with frequency or intensity of electrical mi- 
crostimulation (Stanford et al., 1996; Katnani and Gandhi, 2012) and that peak velocity is attenuated after inactivation of SC (Lee et al., 1988). However, these results only address the distribution of static saccade descriptors, falling short of explaining dynamic properties of the movement (e.g., instantaneous velocity). Another set of studies has advanced the so-called dynamic vector summation model (Goossens and Van Opstal, 2006, 2012), in which the SC controls the desired displacement of the eye through a series of "mini-vectors." Instantaneous control of desired velocity is implicit but not explicitly addressed in this framework.

We tested the hypothesis that SC activity dynamically mediates instantaneous velocity control of saccades. As indicated intuitively, a time-series correlation was first performed by regressing the temporal evolution of SC activity with eye movement profile for each trial, and examining the average of the correlation coefficients. This analysis, by definition, cannot reveal which epoch(s) of the waveforms contributes most significantly to the correlation, and at what transduction time. We addressed these limitations by correlating instantaneous neural activity and eye velocity across trials, an ensemble approach that calculates the correlation between firing rate and velocity on an individual time point basis. Moreover, we focused the analyses only on amplitude-matched movements for each neuron, thus removing confounds of saccade size in the correlation of acrosstrials variability. This strategy determines how individual trial differences are reflected in the SC code, and differentiates itself from previous efforts that correlated neural activity and behavior as the trial progresses in time (Waitzman et al., 1991; Keller and Edelman, 1994; Goossens and Van Opstal, 2006). It also identifies a putative optimal efferent delay between SC activity and eye velocity. We found that instantaneous residual firing rate strongly correlates with instantaneous residual velocity for both within-trial and across-trials analyses. The peak correlation was best aligned with the time of peak eye velocity, and at a population level, the correlation was significant throughout the movement and at a constant efferent delay of $\sim 12 \mathrm{~ms}$. For laminar recording sessions with simultaneously recorded population activity, neurons with the highest firing rates within individual penetrations displayed the strongest correlation. Finally, these relationships were observed not only for ballistic-like, bellshaped velocity waveforms of normal saccades but also for profiles altered by blink perturbations. Thus, individual SC neurons exhibit a code that can control instantaneous eye velocity, akin to how primary motor cortex controls hand velocity (Ashe and Georgopoulos, 1994; Reina et al., 2001; Takei et al., 2018).

\section{Materials and Methods}

Three adult rhesus monkeys (Macaca mulatta, 2 male and 1 female, ages 8,10 , and 12 years, respectively) were used for the study. All procedures were approved by the Institutional Animal Care and Use Committee at the University of Pittsburgh and were in compliance with the U.S. Public Health Service policy on the humane care and use of laboratory animals.

Extracellular spiking activity of SC neurons was recorded as headrestrained animals performed a visually guided, delayed saccade task, with a variable delay of 500-1200 ms, under real-time control with a LabVIEW-based controller interface (Bryant and Gandhi, 2005); task design details can be found in a separate study (Jagadisan and Gandhi, 2017). Neural activity was collected with either a multicontact laminar probe (Alpha Omega; 16 channels, $150 \mu \mathrm{m}$ intercontact distance, $\sim 1 \mathrm{M} \Omega$ impedance of each contact) or a standard tungsten microelectrode (Microprobes, $\sim 1 \mathrm{M} \Omega$ impedance). All electrode penetrations were orthogonal to the SC surface, so that approximately the same optimal vector was encoded across the layers. The saccade target was presented either near the center of the neuron's movement field or at the diametrically oppo- site location. This study reports analyses from 189 neurons, 145 of which were collected with a laminar probe across 18 sessions, and the remaining 44 neurons were recorded with a single electrode (Jagadisan and Gandhi, 2017). All neurons can be classified as visuomotor or motor neurons according to the criterion that activity in the visual epoch $(100-250 \mathrm{~ms}$ following target onset) or the premotor epoch ( -100 to $50 \mathrm{~ms}$ around saccade onset) be significantly greater than baseline activity (Wilcoxon rank-sum test, $p<0.01$ ) (Jagadisan and Gandhi, 2016).

Blink perturbation data were only available for 50 neurons, 43 of which were studied with the single electrode setup and the remaining 7 from a single laminar electrode session. On $\sim 15 \%-20 \%$ of the trials, an air-puff was delivered to one eye to evoke the trigeminal blink reflex. The puff was timed to induce a blink around the time of saccade onset or even trigger the eye movement prematurely. In this case, blink-triggered saccades provide a valuable control against spurious correlations, as the velocity profile of the saccade is altered compared with that of a normal movement and endpoint accuracy is preserved. Thus, if SC dynamically controls the kinematics of the saccade, the perturbed velocity waveforms should be predicted by the SC activity as well. For full disclosure, the data from these 50 neurons are the same as those reported in a previous publication (Jagadisan and Gandhi, 2017). The key distinction is that the previous study assayed SC activity during the saccade preparation phase, and now the focus is on the perisaccade period.

Eye and eyelid movements were detected using the magnetic search coil method. Spike trains were converted to a spike density waveform by convolution with a $5 \mathrm{~ms}$ Gaussian kernel. All movements were aligned on saccade onset. Standard velocity criteria were used to detect the onset (30 $\mathrm{deg} / \mathrm{s})$ and offset (20 deg/s) of normal saccades. For blink-triggered movements, the onset of saccadic component was estimated as the time of a deviation from a spatiotemporal template of a blink related eye movement induced during fixation (Katnani and Gandhi, 2013). The movement profiles were then represented as radial velocity, in which positive values indicate motion toward the movement field and negative values away (Jagadisan and Gandhi, 2017); this differs from the common method in which vectorial velocity representation is always positive and independent of the ideal saccade path toward the target. In other words, vectorial velocity is simply the hypotenuse of the $x$ and $y$ velocities of the eye movement, whereas radial velocity tells us the speed at which the visual axis is moving toward or away from the cell's movement field. This distinction is vital, as we were not able to observe the effects described later when using traditional vectorial coordinates. To remove potential confounds of saccade amplitude on correlations between spiking activity and radial velocity, we additionally limited each neuron's dataset to movements within $\pm 5 \%$ of the mean amplitude. Range of mean amplitude across all neurons spanned 8-25 degrees (median: 12 degrees); and for the analysis comparing normal and blink-perturbed movements, at least 20 trials were available for each condition. All 189 neurons passed the inclusion criteria, 50 of which also had amplitude-matched blink perturbation trials. This pruning yields amplitude-matched movements that still exhibit trial-by-trial variability in their velocity profiles, which constitute the key data for correlation with the corresponding SC activity.

All computations were performed using MATLAB 2016b (RRID: SCR_001622). We started with temporal waveforms of eye velocity and corresponding neural activity for each cell (Fig. 1A). We then subtracted the session's mean velocity and spike density waveforms from each trial data to obtain the respective residuals (Fig. $1 B$ ). This important step removes spurious correlations from generally similar shapes of SC activity and saccade velocity. For within-trial analysis, a Pearson's correlation was determined for each trial's residual velocity and neural activity waveforms. The two residual vectors were shifted relative to each other from $100 \mathrm{~ms}$ to $-100 \mathrm{~ms}$ in $1 \mathrm{~ms}$ increments, and the Pearson's correlation was calculated for each delay $(\Delta t)$. A zero delay indicates that both vectors are aligned on saccade onset. Negative delays signify instances when the neural activity preceded the velocity, which we refer to as an efferent delay (ED). This analysis was performed for every residual neural activity-velocity waveform pairing for each trial of each cell. For acrosstrials analysis, we created a vector of activity residuals at time $t$ and a corresponding vector of velocity residuals at time $t+\Delta t$, where the length of the vectors equals the number of trials. The Pearson correlation 
A
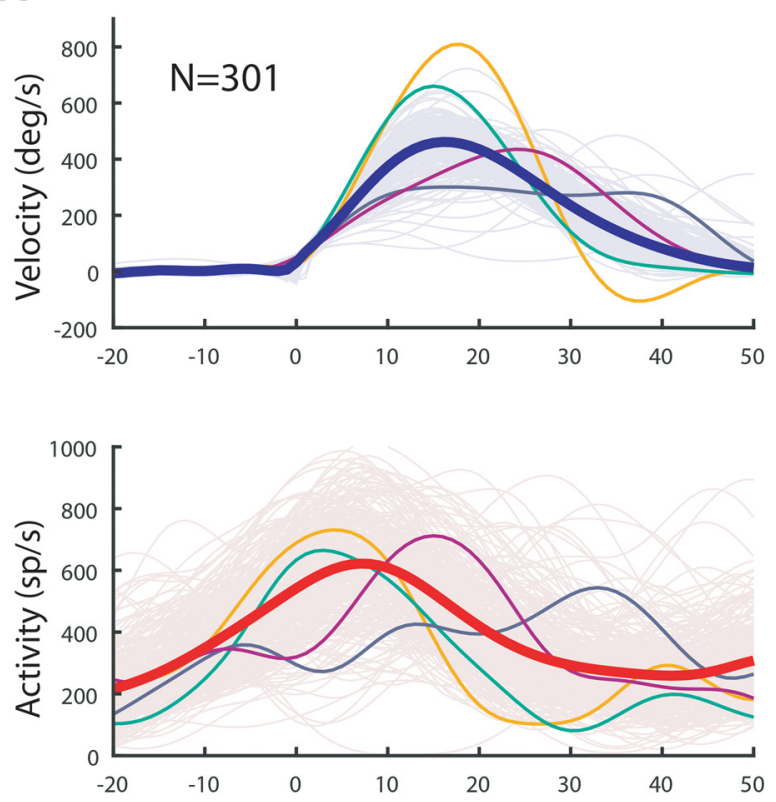

Time from saccade onset (ms)

C

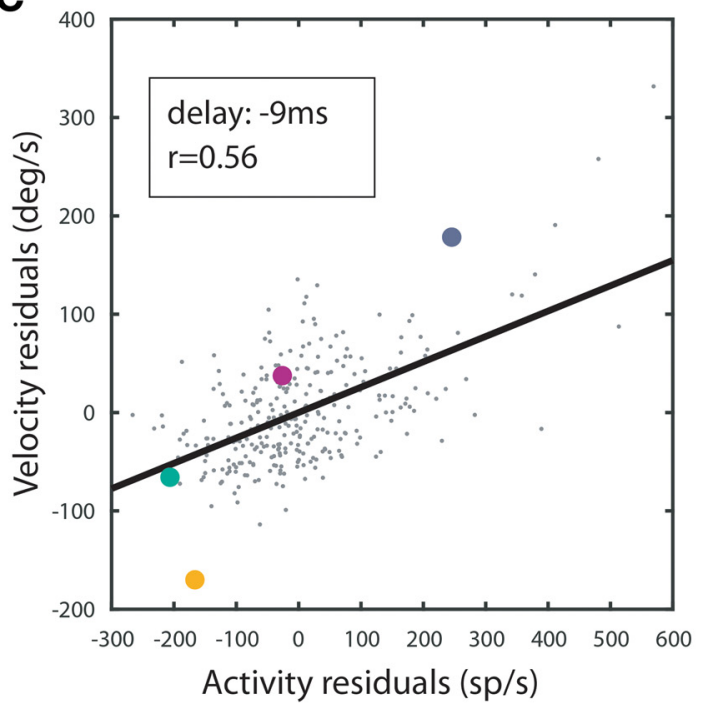

B
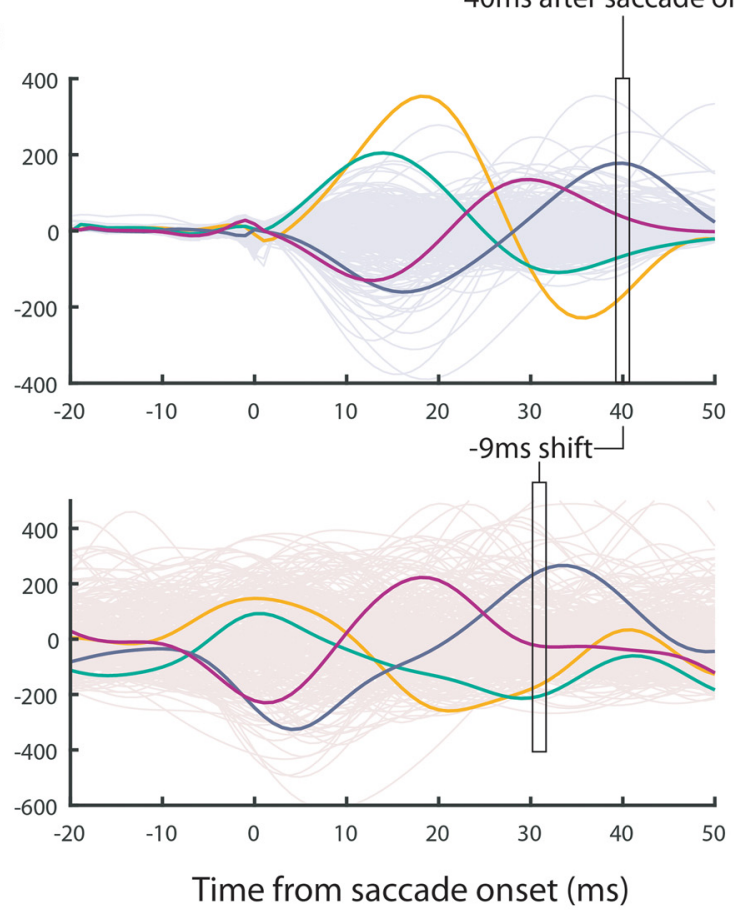

D

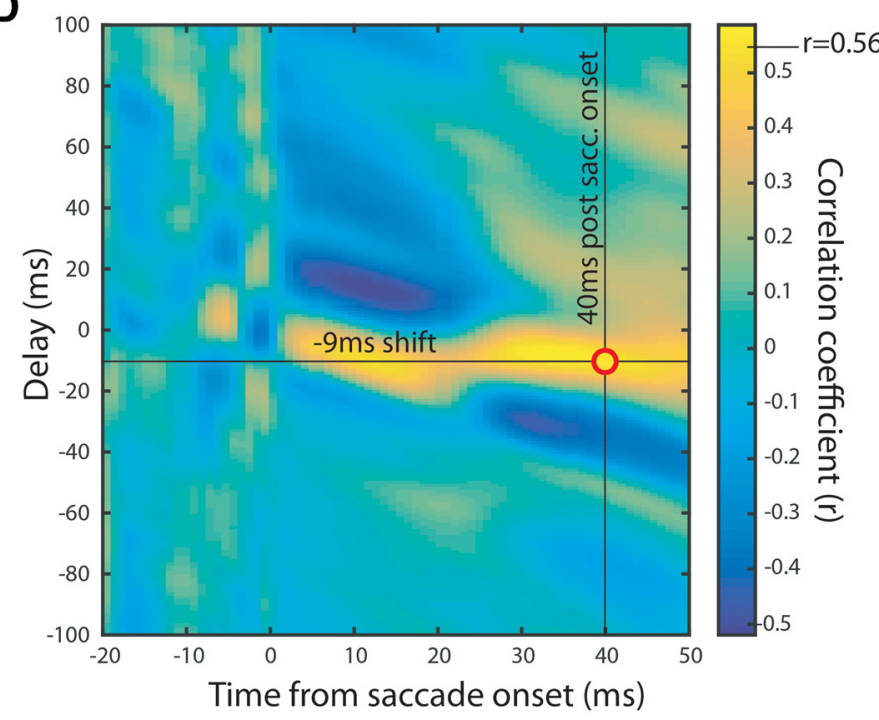

Figure 1. Illustration of across-trial analysis for normal saccades. $\boldsymbol{A}$, Radial velocity traces (top) and their corresponding neural activity (bottom) from one example cell. Positive velocity values represent instantaneous eye movement toward the target, whereas negative values represent movement away. Four traces are highlighted in color to illustrate the fluctuations of individual trials around the mean (thick traces). $\boldsymbol{B}$, Same traces after subtraction of the mean waveform. Boxed sections represent samples of activity and velocity used for regression. Although these samples were analyzed at various delays, only $-9 \mathrm{~ms}$ delay is shown here. $\boldsymbol{C}$, Velocity samples from box in $\boldsymbol{B}$ are plotted as a function of their corresponding activity sampled at a $-9 \mathrm{~ms}$ delay. Regression line and the correlation coefficient are provided. D, Correlation coefficient values (color) represented as a function of the delay and time point of the saccade.

between these two vectors was determined (Fig. 1C). We repeated this procedure for every time point in the saccade and for every $1 \mathrm{~ms}$ shift, resulting in a correlation coefficient for each combination of time relative to saccade onset and delay (Fig. 1D). This technique allowed us to examine the SC effects on eye velocity at every time point of the saccade in $1 \mathrm{~ms}$ resolution. We repeated both correlational analyses on blink-perturbed trials to determine whether the results persist even when the saccade properties are altered.

Experimental design and statistical analyses. To determine the significance of the results of within-trial analysis, we randomly paired a residual velocity trace of one trial with residual activity data of another trial from the same session's data and determined the Pearson correlation for the span of delay values. For across-trials analysis, we randomly shuffled the order of elements in each residual vector (instantaneous across-trial shuffle) before determining the correlation coefficient. The shuffling procedures were repeated 100 times for both types of analyses. Deviations of unshuffled correlation results outside 2 SD bounds generated from shuffled data were deemed statistically significant.

To further validate that the influence of the SC on velocity continues throughout the entirety of the saccade, we calculated the duration of significant correlation for each cell. To do this, we determined the confidence threshold by performing the across-trial analysis on shuffled data for each cell 100 times. Then we determined that the correlation was significant at those points where the real correlation exceeded 2 SDs 

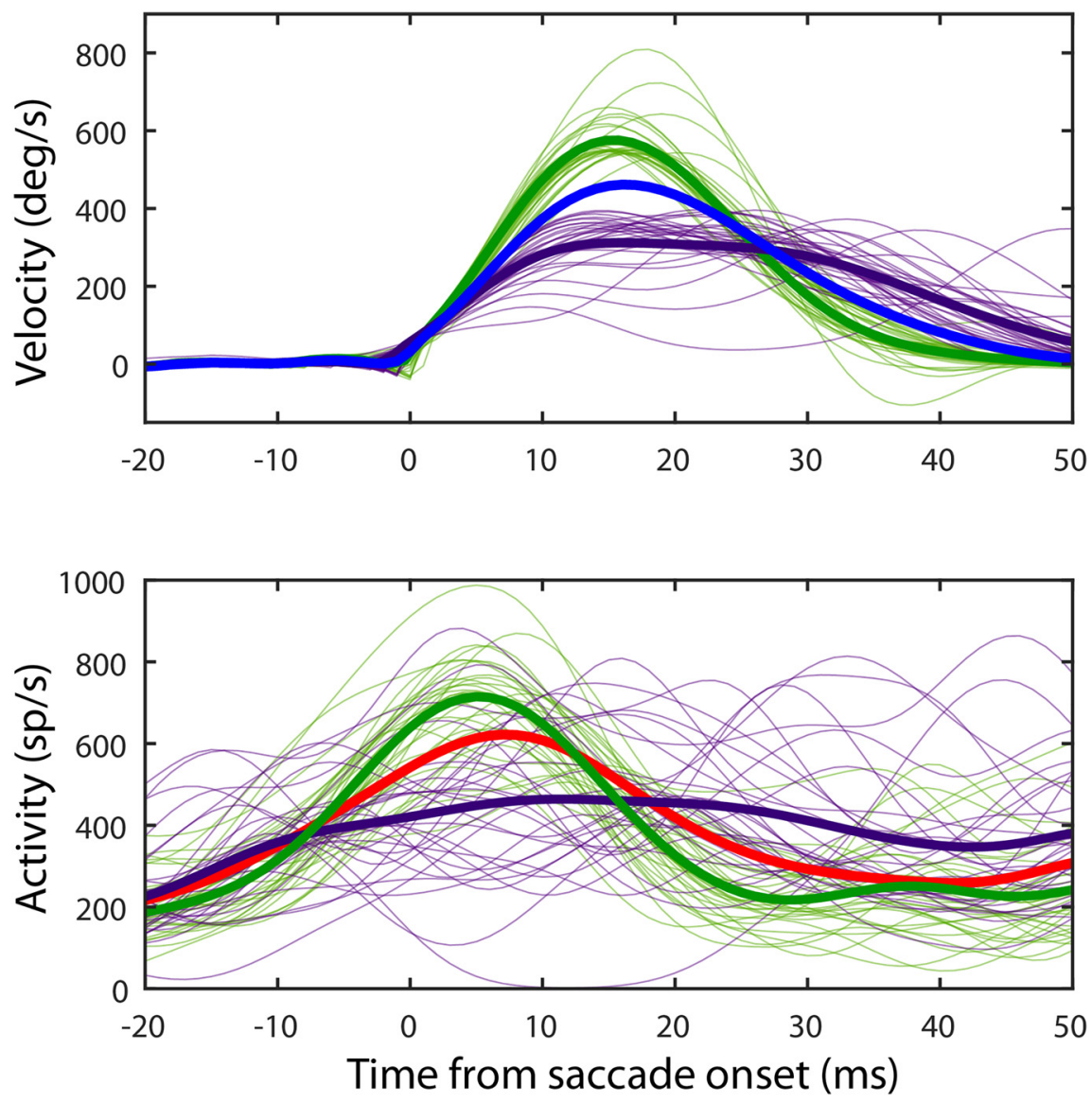

Figure 2. Temporal profiles of eye velocity and neural activity for normal saccades. Green traces represent the $10 \%$ of trials with highest peak velocity. Purple traces represent the $10 \%$ of trials with lowest peak velocity. Thick, matching-color traces in each subplot represent averages. Blue and red traces are from Figure $1 A$. Data are from one session. In addition to the general similarity of waveforms between the velocity (top) and their corresponding activity (bottom), there is also a correlation between the temporal features. Traces with high (low) peak velocity and short (long) deceleration duration are associated with similar profiles in the neural activity.

around the mean of the shuffled results. Summing the instances at which correlation was significant gave us the total duration of the correlation.

In a separate analysis, we looked at the subset of data that was collected using laminar probes. This subset allowed us to examine the effect of depth of SC neurons on our results. The number of channels with neural data ranged from 4 to 16 per session. Because each session did not have enough channels for sufficient statistical power, we de-meaned the data from each session and combined all channels. We then used linear regression on the de-meaned correlation coefficients of these channels and the corresponding firing rate to establish a trend.

\section{Results}

Figures $1 A$ and Figure 2 show temporal profiles of saccade velocity for many amplitude-matched movements and corresponding spike density signals for one SC neuron. The saccade closely matched the neuron's preferred vector. A within-trial correlation can be readily appreciated by the similar bell-shaped profiles of both velocity and activity waveforms. This relationship can be intuitively queried by correlating the firing rate with velocity for different transduction delays. Figure $3 A$ plots the results of such within-trial analysis for normal saccades across all 189 neurons in our database. The mean peak Pearson correlation $(r=0.179)$ was observed for an efferent delay (ED) of $13 \mathrm{~ms}$, equivalently $\Delta t=$ $-13 \mathrm{~ms}$. The correlation was significantly different from the pattern observed for shuffled data. This result therefore indicates that the residuals of both SC activity and eye velocity fluctuate around the mean in a coherent fashion and that SC activity can influence saccade velocity.

A major limitation of the within-trial analysis is that it offers no information about the correlation at each instant during the saccade. The correlation coefficient could peak if any sufficiently long sequence of the activity correlates with the corresponding sequence in velocity. One can imagine that SC could, perhaps, encode only the accelerating phase of the eye movement. The deceleration phase could be unaffected by SC activity and instead be guided by muscle viscoelastic properties. In this case, the correlation would peak at a particular ED because the first half of both signals is correlated, but would provide little evidence to support our hypothesis that SC dynamically influences the entire saccade.

Figure 2 illustrates that a closer inspection reveals a hint of temporal control of eye velocity. The top $10 \%$ of trials with highest peak velocities exhibited short deceleration durations, and the associated activity profiles exhibited robust bursts that peaked early in the saccade followed by rapid reduction in firing rates (green traces). In contrast, the $10 \%$ of trials with lowest peak velocities, which had long deceleration phases, matched with muted peak rates that continued for prolonged periods (purple traces). Such trends warrant a systematic examination of acrosstrials analysis to determine precisely the time course of correlation between activity and velocity (for details, see Fig. 1; Materials and Methods). Assembled across trials, the residual firing rates were regressed against residual eye velocities separated by a delay. This procedure was repeated for a large range of delays and for all time points of a saccade. Figure $3 B$ shows the correlation coefficients for all combinations of saccade time points and ED values. A horizontal band of high correlation values is noted for the duration of the saccade for an ED of $12 \mathrm{~ms}$. The correlation values in this band (Fig. 3C) are even higher (peak: $r=0.278$ ) than that found in within-trial analysis. Results from the across-trials analysis therefore provide stronger evidence that SC dynamically influences eye velocity throughout the entire saccade. Additionally, it is prudent to mention that this analysis is identical whether performed on residuals or unaltered data, thus providing a more direct evidence of correlation compared with the within-trials analysis.

Next, we explored the temporal characteristics of instantaneous activity-velocity correlations. We found that the time of peak correlation was well aligned with the time of peak velocity, after accounting for the efferent delay for each neuron (Fig. 4A), although a substantial number of neurons exhibited their strongest correlations before or after peak velocity (negative or positive values on the $x$ axis, respectively). A paired $t$ test could not reject the null hypothesis that the mean difference was zero $(p=0.15)$. In contrast, the extent of SC's influence over saccade velocity, measured as a proportion of saccade duration, reveals a flat dis- 

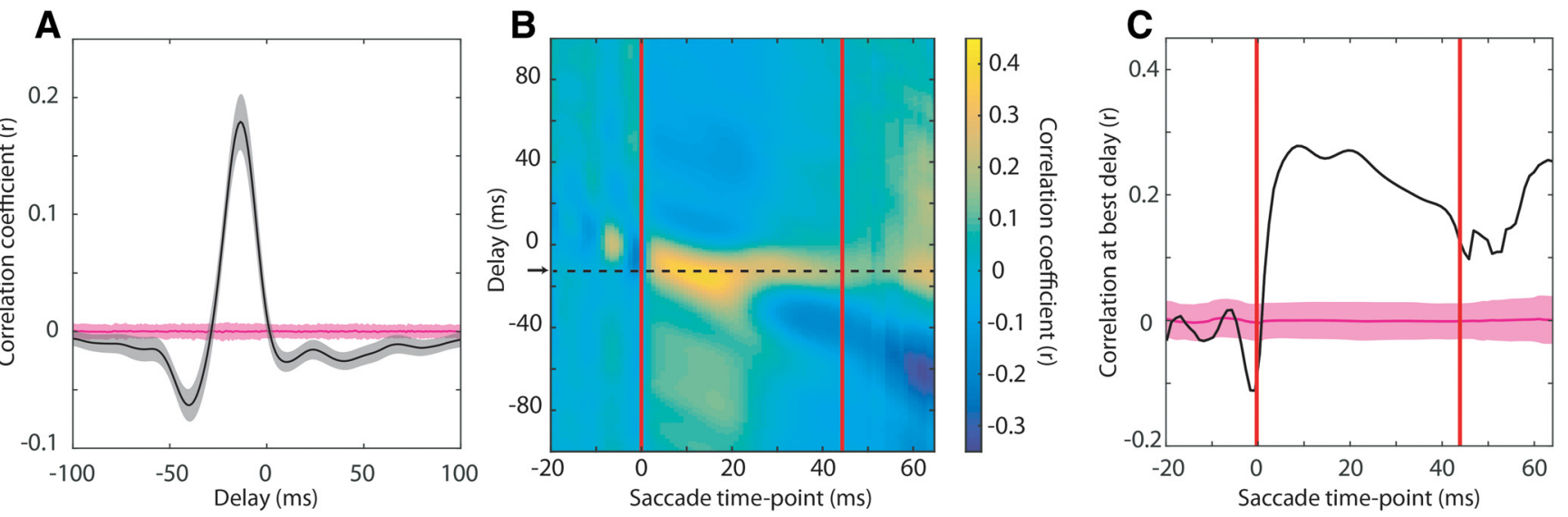

Figure 3. Summary of within- and across-trial correlation analyses for normal saccades. $\boldsymbol{A}$, Within-trial correlation analysis. Black line indicates the correlation coefficient between activity and velocity residuals as a function of the temporal shift. Gray outline represents $2 \mathrm{SEs}$ around the mean patterns from 189 neurons. Pink line and outline represent the mean correlation coefficients and 2SDs from the mean of the shuffled data. $\boldsymbol{B}$, Across-trials correlation analysis. Heatmap of correlation coefficients between SC activity and eye velocity residuals for each time point during the saccade and temporal shift between the two residual vectors ("delay"). Arrow and horizontal hyphenated line represent the efferent delay ( $-12 \mathrm{~ms}$ ) at which the average correlation was highest. Left and right vertical red lines indicate the beginning and end, respectively, of the shortest saccade in the dataset. Data past the rightmost red bar exclude saccades that terminated before the time points on the $x$ axis. $\boldsymbol{C}$, Correlation coefficients as a function of saccade time points for the optimal efferent delay shown in $\boldsymbol{B}$. Pink line and outline represent the mean and 2 SDs for the across-trials analysis performed on shuffled data.

A

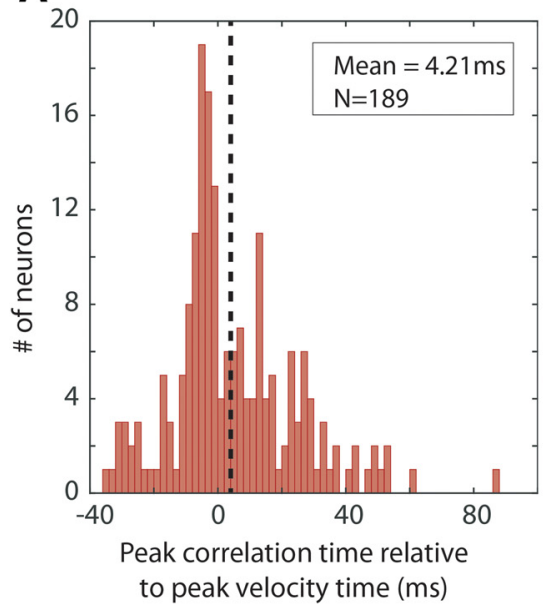

B

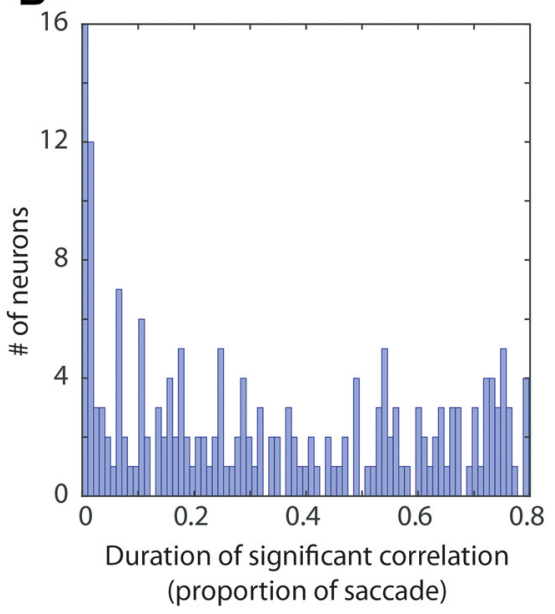

C

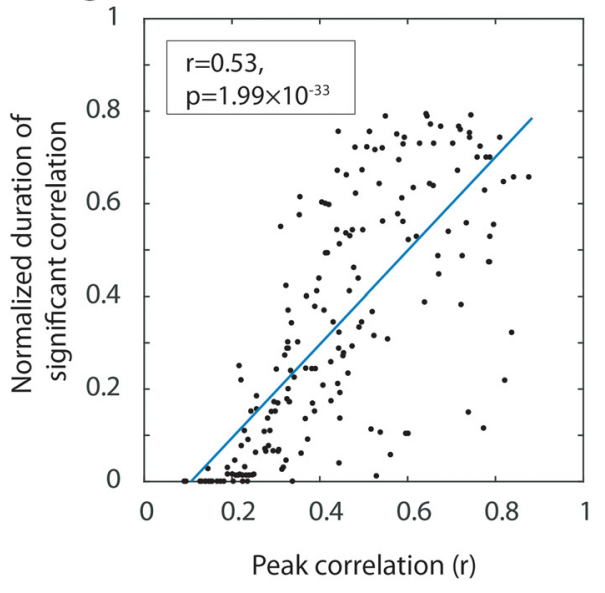

Figure 4. Temporal characteristics of activity-velocity correlation. $\boldsymbol{A}$, Histogram of average peak correlation time relative to average peak velocity time for each neuron. The count on $y$ axis indicates the number of neurons. $\boldsymbol{B}$, Histogram of cumulative duration (as proportion of total saccade length) for which the correlation remained above significance level. $\boldsymbol{C}$, Relationship between peak correlation and the duration of the correlation. Each point indicates one neuron. Blue line indicates the best fit line to the data.

tribution (Fig. 4B). Further, cells that had a shorter duration of significant correlation tended to have a lower peak correlation (Fig. $4 C ; r=0.53, p=1.99 \times 10^{-33}, F$ test). The relationship between degree and duration of influence could be explained by cells with higher correlation values having a higher likelihood of rising above the significance level over time. Thus, SC neurons tend to exhibit most influence over eye kinematics around the peak of the saccade velocity profile, and those cells that showed a higher peak correlation continued to influence eye velocity well past the peak, to saccade completion.

We then identified which properties of the neuronal population contributed to significant correlations. When examining the entire population of cells, simple linear models found no relationship between a cell's peak correlation and its peak firing rate or its location along the rostral-caudal extent of the SC ( $p=0.88$, $F$ test). However, when we considered only the cells from a single laminar recording, we observed an increasing trend between the cell's firing rate and its peak correlation (Fig. 5A). When data from the laminar recordings were pooled by subtracting the average peak firing rate and correlation measures of each session, a statistically significant linear relationship was observed (Fig. 5B; $p=7.4 \times 10^{-6}, F$ test). This suggests that there is a strong relationship between firing rate and instantaneous velocity for neurons along the dorsoventral axis of the SC.

To assess the robustness of the influence of SC activity over instantaneous eye velocity, we turned to the 50 neurons for which we also had blink-perturbation data. Such saccades do not exhibit the stereotypical, bell-shaped profile and therefore offer an opportunity to assess whether the correlation persists, even in the presence of perturbation. Figure 6 illustrates representative data and the analysis approach in the same format as done for control saccades. The same qualitative features can be noted despite the blink-induced perturbation. Figure 7 (top row) displays the within- and across-trial analyses for normal trials in the 50 neurons. The same general trend persisted, even for this subset of neurons. The best EDs for the normal, unperturbed data were 11 
A

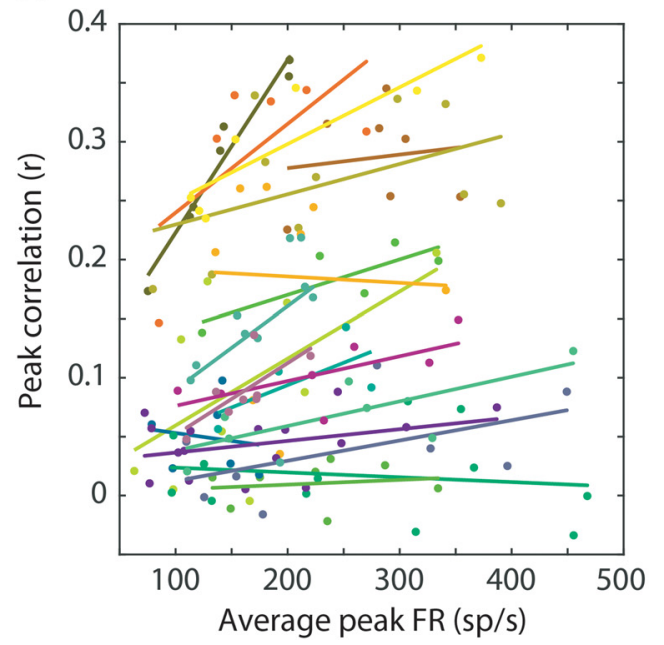

B

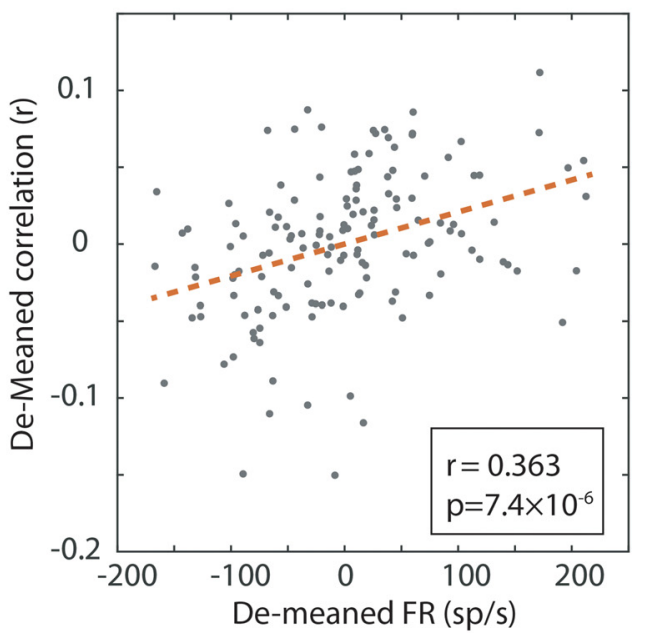

Figure 5. Analysis of data recorded from laminar probes. $\boldsymbol{A}$, Peak correlation of each SC neuron is plotted against the average peak firing rate of that neuron. Neurons recorded in the same penetration are plotted using the same color. Thus, each color represents data from different sessions. The best fit line to each session's data is shown in the matching color. $\boldsymbol{B}$, Data from $\boldsymbol{A}$, de-meaned and pooled across sessions. Each de-meaned value is obtained after subtracting the respective average across all neurons in its track. Red hyphenated line indicates the best fit line.

and $12 \mathrm{~ms}$, and the peak correlations were $r=0.267$ and $r=0.441$ for within- and across-trial analyses, respectively. For the blinkperturbed data from the same neurons, the peak correlations were $r=0.210$ and $r=0.386$ for both within- and across-trial analyses, respectively; and the ED was 13 for both. The correlative relationship was largely intact despite the blink-induced perturbation.

To further characterize the impact of blink perturbation, we computed for each neuron the linear relationship between residual activity and velocity distributions across the duration of the movement. Assuming the data contained $n$ trials, and the average duration of the amplitude-matched saccades is $d m s$, a single regression was performed across $n d$ points. The activity data were time-shifted relative to the velocity distribution to account for the neuron's ED. This was done separately for the normal and blink-perturbed data from each of the 50 neurons. Figure $8 \mathrm{~A}$ shows a scatter plot of the regression slopes for each neuron in the two conditions. Overall, the slopes tended to be greater during the blink condition (paired $t$ test, $p=0.0013$ ). We speculate that reacceleration of saccades in the blink-perturbation condition (Goossens and Van Opstal, 2000; Gandhi and Bonadonna, 2005) produces residuals that likely increase the regression slope. Moreover, we found a strong relationship between each neuron's regression slope and the goodness of fit in both normal $(r=0.72$, $\left.p=2.37 \times 10^{-7}\right)$ and perturbation $(r=0.35, p=0.02)$ conditions (Fig. $8 B$ ). This suggests that, although SC activity is updated to reflect the change in velocity, this compensation is likely not complete and that additional temporal control signals are added downstream in the brainstem burst generator.

A peculiar finding of our analysis is the statistically significant negative correlation observed just before saccade onset for across-trials regressions (Figs. 3C, $7 C$ ). This is not a physiological effect but instead a byproduct of the data processing, which is why we present it at the end of Results. Movement onset is defined as the time point when the absolute velocity exceeds 30 $\mathrm{deg} / \mathrm{s}$, which means that there must be eye motion before reaching this criterion. Consider once again the eye velocity traces in Figure 2. The subset of trials with high (green traces) and low (purple traces) peak velocities actually have the inverse initial velocity relationship just before saccade onset: the green and purple traces exhibit lower and higher initial velocities, respectively.
The underlying neural activity waveforms before saccade onset, however, do not exhibit this reversal before saccade onset, thus producing the negative correlation. This effect is, as expected, not present when the analysis is performed with data aligned on saccade end (data not shown). A comparable explanation accounts for the negative correlation observed for the large negative delays $(\sim-40 \mathrm{~ms})$ of the within-trial analysis (Figs. $3 A, 7 A, F)$. The correlation at such delays is dominated by a regression between the ascending phase of neural activity with the decelerating phase of the eye movement. Of course, these long delays are not reflective of the effective $\sim 12$ ms transduction time from the SC to the eye plant.

\section{Discussion}

We described a previously unreported phenomenon on how SC activity exerts instantaneous control of saccadic eye movements. We found a strong correlation between the motor burst of SC neurons and eye velocity for an efferent delay of $\sim 12 \mathrm{~ms}$. The correlation was noted for both within-trial and across-trials analyses. The latter approach, in particular, demonstrated that the correlation remained high for the duration of saccade, lending support for SC control of instantaneous eye speed. It revealed a robust distributed population coding scheme reminiscent of a synfire chain (Diesmann et al., 1999; Shmiel et al., 2006), wherein individual neurons exert influence over saccade dynamics sequentially at different times, collectively spanning the duration of the saccade. Comparable correlation structure and ED were also observed for blink-perturbed movements, whose velocity profiles deviate significantly from the stereotypical bell-shaped waveforms (Goossens and Van Opstal, 2000; Gandhi and Bonadonna, 2005), although it was particularly important to project the velocity vector onto the preferred vector of the SC neuron (Jagadisan and Gandhi, 2017). We also learned that, within individual electrode penetrations, cells with higher firing rates exhibited a stronger correlation with velocity. This suggests a velocity-activity relationship based on the depth of each cell as well as its spiking properties. Finally, we uncovered the interesting feature that regressions with the largest correlations also had the biggest slopes, implying that eye velocity is 
A
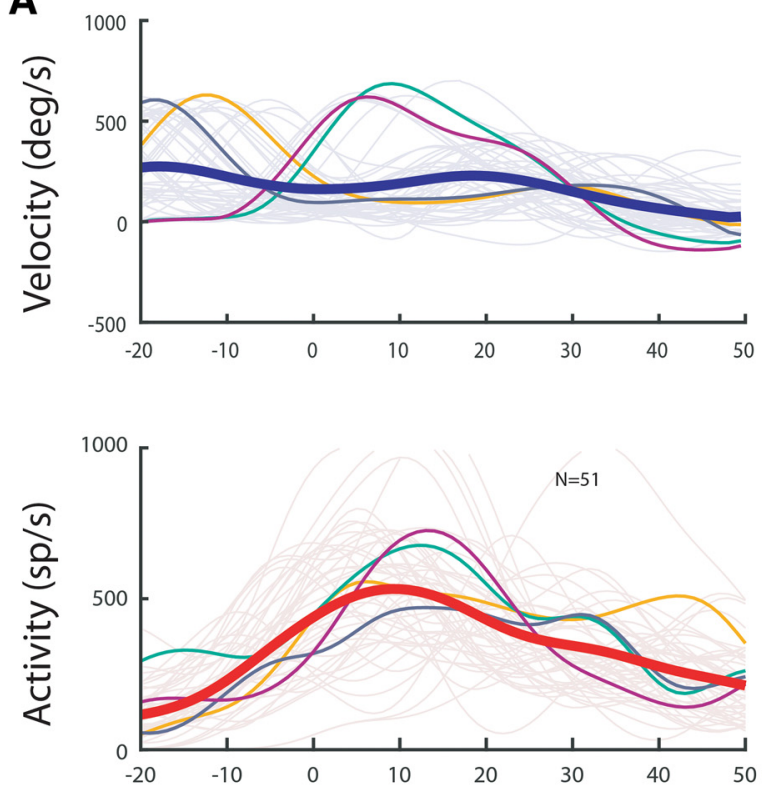

Time from saccade onset (ms)

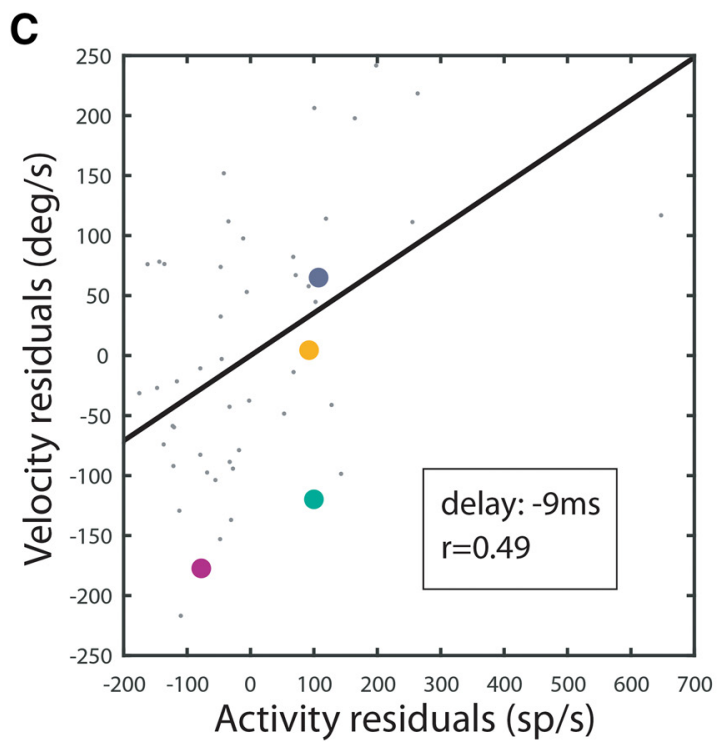

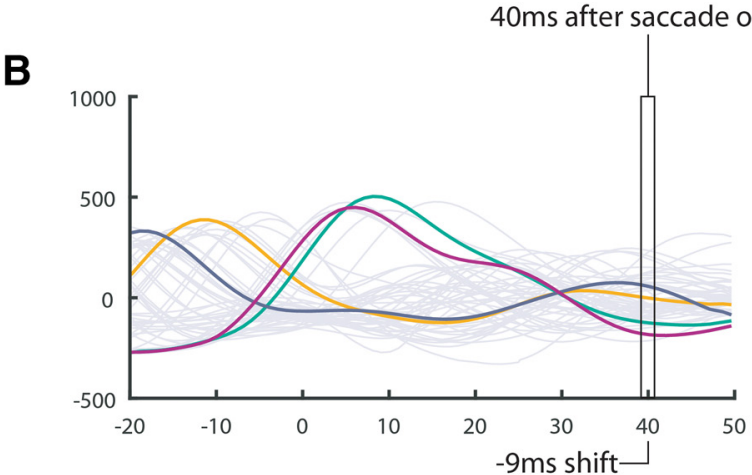

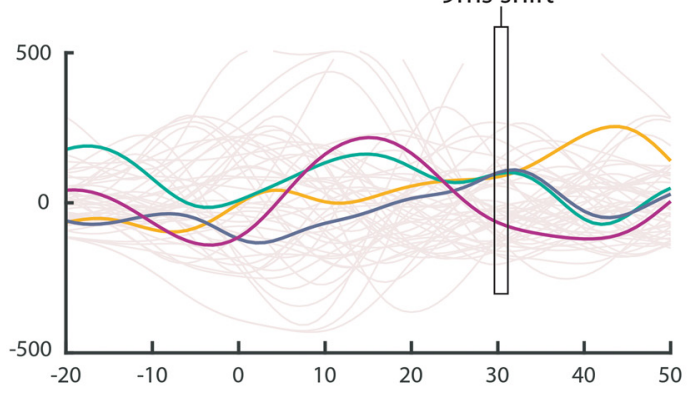

Time from saccade onset (ms)

\section{D}

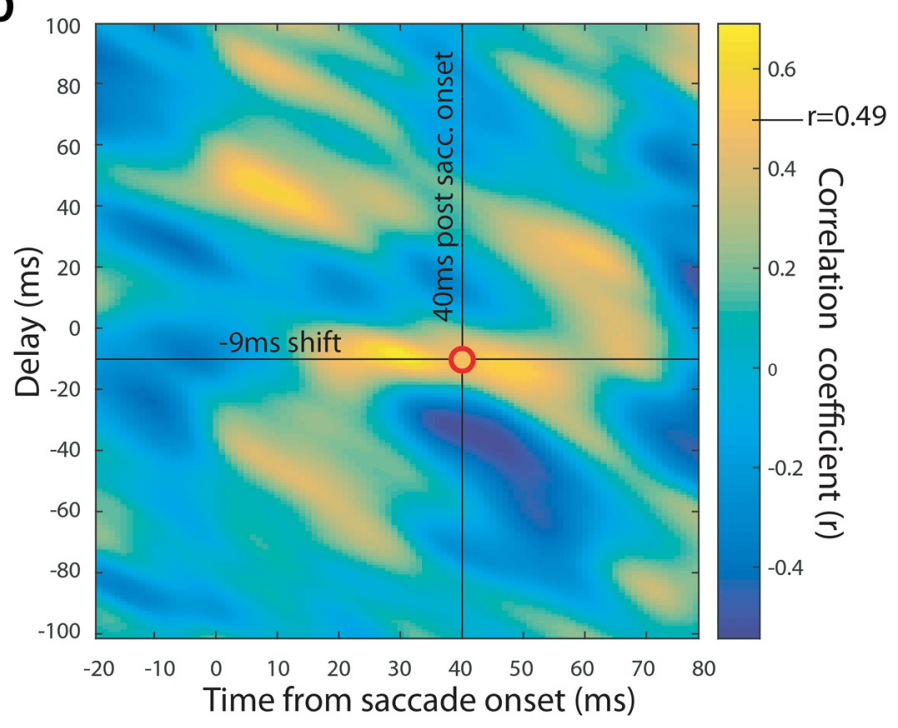

Figure 6. Illustration of across-trial analysis for blink-perturbed saccades. Same format as in Figure 1. A, Velocity and spike density traces. $\boldsymbol{B}$, Residuals of traces in $\boldsymbol{A}$. C, Correlation between velocity and activity residuals at $-9 \mathrm{~ms}$ delay. $\boldsymbol{D}$, Heatmap of correlations at every delay and at every saccade time point.

more sensitive to firing rates of those neurons that better account for its variance.

While several models of saccade control describe static features of SC activity most likely to influence a saccade, they rarely describe the time course of such influences. The across-trials analysis in our study provides a direct estimate of the efferent delay at which SC activity is most likely to influence the observed movement. The efferent delay is indicative of the transduction time of neural signals from the SC to the extraocular muscles. Studies of SC stimulation have established a 25-30 ms latency for movement initiation (Stanford et al., 1996; Katnani and Gandhi, 2012) and a shorter 10-12 ms delay for influencing an ongoing movement (Miyashita and Hikosaka, 1996; Munoz et al., 1996;
Gandhi and Keller, 1999). The ED that yielded the strongest correlation from our neural recording data was $\sim 12 \mathrm{~ms}$. Crucially, it remained relatively constant throughout the movement, although we did observe a broader range of ED values with higher correlation coefficients around saccade onset (Fig. 3C). Although the ED values are different for microstimulation and recordings studies, a direct comparison should be avoided because the underlying network-level processes associated with movement preparation, which are implicitly incorporated in neural activity, are likely disengaged when microstimulation is used to trigger a saccade.

We are intrigued by the observation that peak correlation between activity and velocity increased as a function of peak firing 

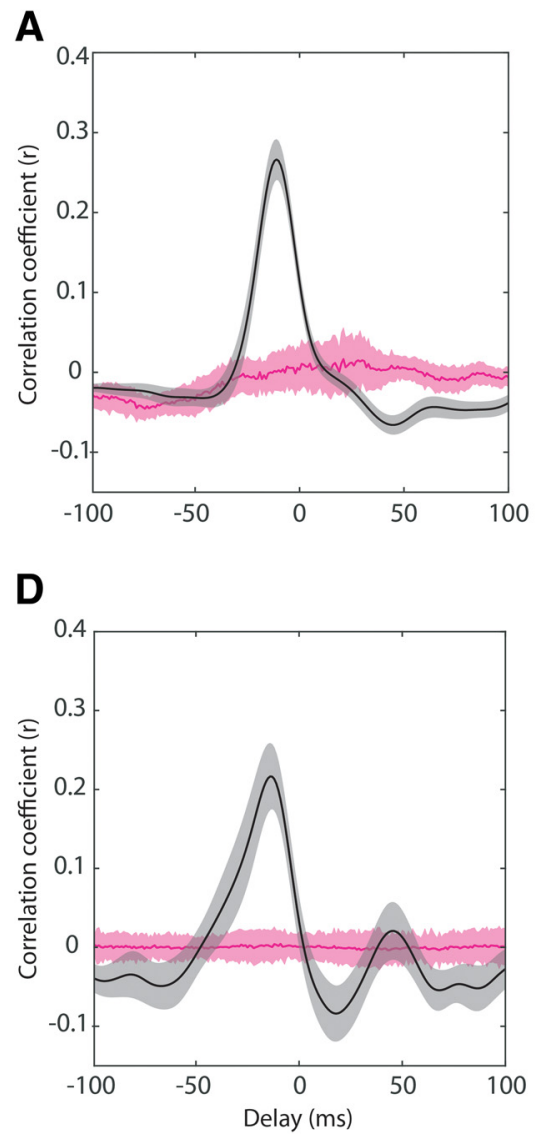

B

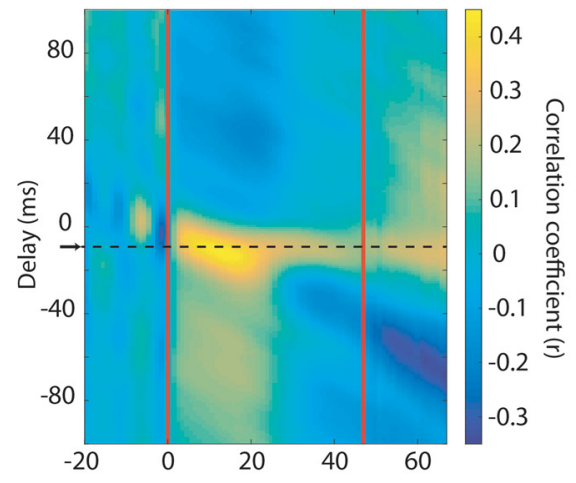

E

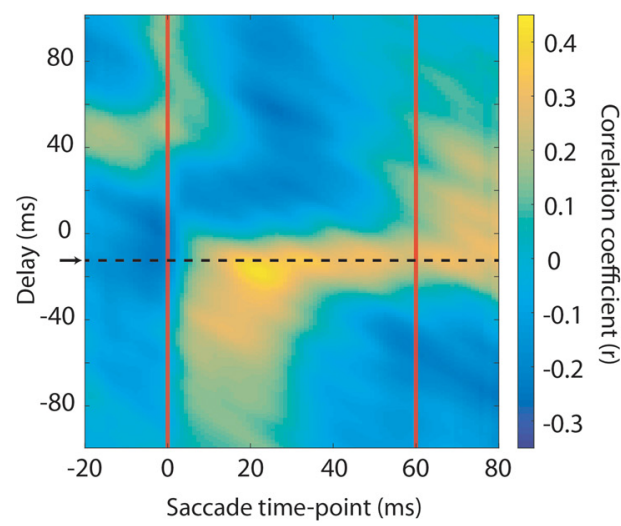

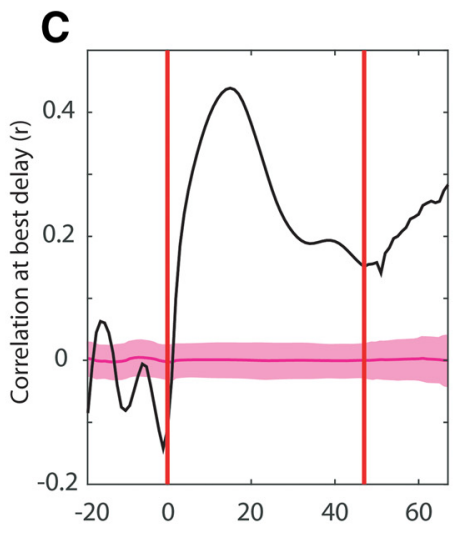

$\mathbf{F}$

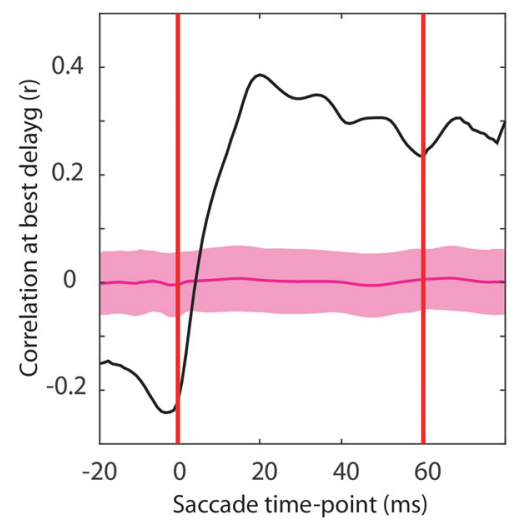

Figure 7. Comparison of correlation analyses for blink-perturbed and normal saccades. Within-trial correlation between activity and velocity residuals for (A) normal and (D) blink-perturbed saccades available for 50 of 189 neurons. The heatmaps of correlation coefficients obtained from across-trials analysis for $(\boldsymbol{B})$ normal and $(\boldsymbol{E})$ blink-perturbed movements. Correlation coefficients as a function of saccade time points for the optimal efferent delay for $(\boldsymbol{C})$ normal and $(\boldsymbol{F})$ blink-perturbed saccades. The plots follow the same conventions used in Figure 3.

A

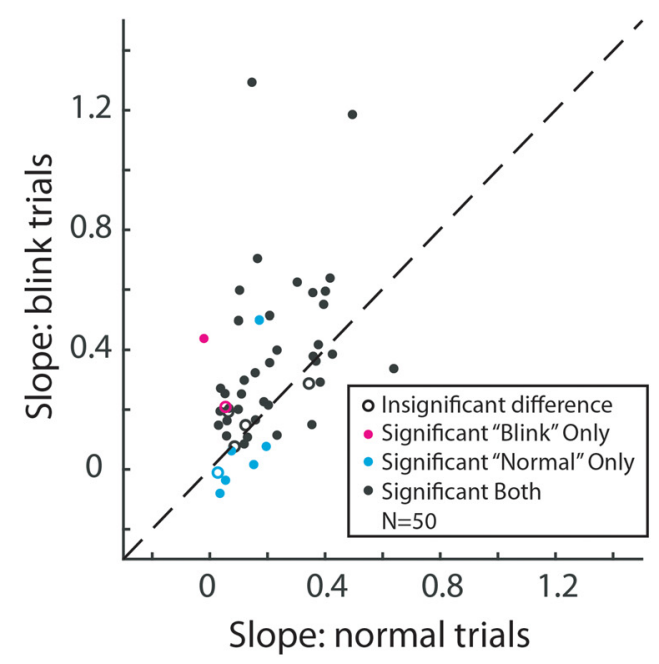

B

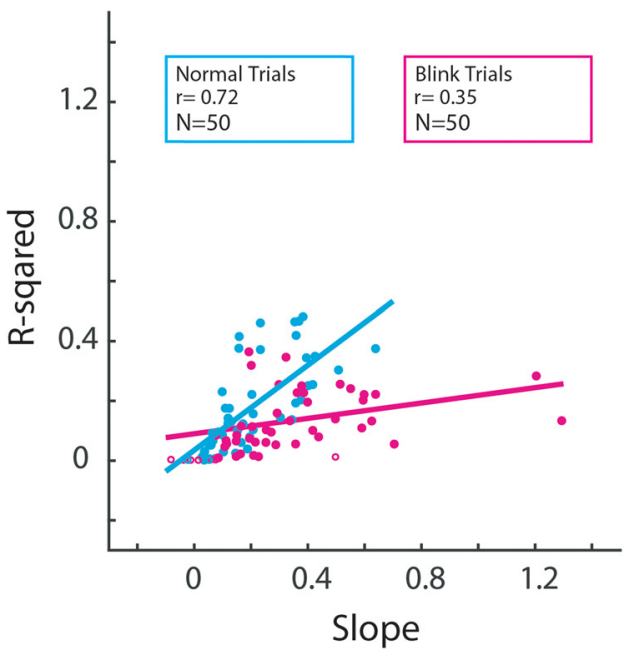

Figure 8. Linear regression features between SC activity and eye velocity. A, A pairwise comparison of the regression slopes obtained for normal ( $x$ axis) and blink-perturbed (y axis) conditions for each neuron. Slopes statistically significant different from zero for normal-only, blink-only, and both types of trials are shown in cyan, magenta, and black colors, respectively. Filled (open) circles denote neurons for which the slopes for normal and blink conditions were (not) statistically significantly different from each other. Hyphenated line indicates the unity relationship. $\boldsymbol{B}$, Relationship between slope and $R^{2}$ values. Cyan represents normal trials. Magenta represents blink perturbation trials.

rate, but this effect was most notable for neurons within individual electrode tracks and not across the entire SC (Fig. 5A). We think this is important because firing rate can vary both as a function of topographic location on the SC map, as well as dor- soventrally, and the influence of a control signal must ideally not be dependent on topography (i.e., the amplitude and direction of the saccade being executed). Analyses from separate, unpublished work in our laboratory indicate that peak firing rate of the 
motor burst changes nonlinearly with depth, reaching a maximum in the intermediate layers and decreasing gradually for dorsal and ventral locations. Neurons with the highest firing rates resemble the classical saccade-related burst neurons, some with buildup activity, and are likely those that project to the gaze centers in the brainstem (May, 2006; Rodgers et al., 2006).

Previous studies have addressed relationships between neural activity and movement parameters in several ways. For SC control of saccades, the focus has been on static parameters. For example, weaker bursts of activity produce saccades with lower peak velocity (Edelman and Goldberg, 2001), peak velocity is correlated with frequency or intensity of microstimulation (Stanford et al., 1996; Katnani and Gandhi, 2012), and peak velocity is attenuated after inactivation of SC (Lee et al., 1988). Modest correlations with SC activity have also been reported for head movements (Walton et al., 2007; Rezvani and Corneil, 2008) and electromyographic activity in proximal limb muscles (Stuphorn et al., 1999). Fewer studies have correlated the temporal profiles of neural activity and eye movements for individual trials or an average across trials (e.g., Waitzman et al., 1991; Keller and Edelman, 1994; Goossens and Van Opstal, 2006), but such analyses do not determine which epoch(s) of the movement are controlled. We employed an additional strategy and computed correlations on across-trials variability of neural activity and eye velocity. By performing the correlation in $1 \mathrm{~ms}$ increments during a saccade, we gained insights into instantaneous control without being constrained by the similarity of the temporal features of the two signals. The sliding temporal analysis of across-trials variability affords the ability to infer the efferent delays in communication between SC and extraocular muscles, unlike the aforementioned studies, and the consistency in the estimated delay values speaks to the robustness of the results. Furthermore, by matching saccade amplitude and minimizing endpoint variance, the analysis is able to better capture both moment-by-moment and trial-by-trial variabilities in the signals. Such analyses have been used in skeletomotor research, with significant correlations identified between neural activity waveforms in cortical areas and hand velocity profiles (Ashe and Georgopoulos, 1994; Reina et al., 2001; Takei et al., 2018). Trial-to-trial variability in eye and hand velocity has also been attributed to variability in neural activity occurring earlier in the trial, for instance, during sensory input (Osborne et al., 2005; Huang and Lisberger, 2009) and motor preparation (Churchland et al., 2006a; Jagadisan and Gandhi, 2017), but this perspective precludes insight into direct dynamic control during ongoing movements.

Our study provides evidence consistent with the hypothesis that SC neurons encode instantaneous saccade velocity. This finding does not conform readily to its standard role in spatiotemporal transformation, that the spatial distribution of population activity in the deeper layers determines the saccade vector and that downstream structures generate the firing patterns that reflect the velocity profile of the eye movement. The result that SC activity does influence peak saccade velocity (cited above) somewhat aligns to a modified framework, the dual-coding hypothesis (Sparks and Mays, 1990), in which the level of SC activity acts as a gain factor on the brainstem burst generator (Nichols and Sparks, 1996). However, influencing saccade speed through a global gain is not equivalent to impacting instantaneous motion. Our results agree more with a dynamic vector summation algorithm (Goossens and Van Opstal, 2006, 2012) in which the SC controls the desired instantaneous displacement of the eye, although this model abstains from making direct statements about instantaneous velocity control, and it also incorporates a spatio- temporal transformation component. Another framework, which does not use a spatiotemporal transformation between the SC and lower brainstem, places the duty of dynamic control on the cerebellum, and the SC has secondary roles of providing a directional drive and triggering the movement (Quaia et al., 1999). We do not believe the instantaneous correlation between velocity and neural activity we report here follows from this model, but proponents could render it an epiphenomenon.

\section{Revised view of neural control of saccades: a speculation}

All extant models of saccade control stipulate the SC output to be a desired displacement signal, which is in position coordinates. How does the notion that SC encodes a velocity signal impact such models? We start by first describing the core elements of an existing framework (Fig. 9A). The discharge rate of the active population of SC neurons is decoded into a desired displacement signal $\left(\Delta E_{d}\right)$ through a mechanism, such as weighted vector averaging or summation (for review, see Gandhi and Katnani, 2011). This signal is the primary input to the comparator of an engineering-inspired, local feedback loop (Robinson, 1975; Jürgens et al., 1981) that subtracts the instantaneous eye displacement $(\Delta E)$ from $\Delta E_{d}$ to compute a dynamic motor error (m.e.), which in turn drives pontine excitatory burst neurons (EBNs). The firing rate of EBNs is a nonlinear function of motor error and reflects an eye velocity signal $\left(E_{E B N}^{\prime}(t)\right)$ (Van Gisbergen et al., 1981) that is further processed downstream by the nucleus prepositus hypoglossi (NPH) and extraocular motoneurons to generate the saccade. The velocity signal from the EBNs must be integrated into position coordinates to produce the $\Delta E$ signal needed at the comparator. Crucially, $\Delta E$ must be reset to zero after each saccade so that the model can generate an accurate movement when the next $\Delta E_{d}$ signal is presented by the SC. Thus, the local feedback branch includes a resettable neural integrator. Finally, the omnipause neuron (OPN) gates the saccadic system by inhibiting the EBN (Keller, 1974). The high-frequency burst of SC neurons removes the OPN gate through disynaptic inhibition (Yoshida et al., 2001), which allows the local feedback loop to initiate and control the saccade dynamics until motor error reduces to zero, when the OPN once again inhibits the EBN. This framework, originally proposed by Robinson (1975), has been tremendously influential, in part because predicted activity profiles by various nodes of the circuit were observed in brainstem neurons in later experiments. However, neural correlates of two essential elements of this circuit, the resettable neural integrator and the comparator (both denoted in red), have remained elusive.

To consider the possibility that SC outputs a velocity signal, we offer the speculative framework shown in Figure 9B. The centerpiece is the spatiotemporal distribution of population activity in SC neurons. The Gaussian-like SC population response, in both space and time, produces the stereotypical bell-shaped velocity waveform. We suggest that the intrinsic circuitry in SC, particularly the balance of excitation and inhibition, is essential for molding both the spatial and temporal components of the population response (Kaneda et al., 2008; Phongphanphanee et al., 2014). We speculate that the burst profile effectively also mediates burst duration and that the SC network exhibits refractory dynamics that account for the resettable neural integrator effect (Kustov and Robinson, 1995; Nichols and Sparks, 1995; Schlag et al., 1998). Of course, some of these network features could also be mediated by biophysical properties of SC neurons, which could be implemented through a spiking neural network framework (Morén et al., 2013; Kasap and van Opstal, 2017). 

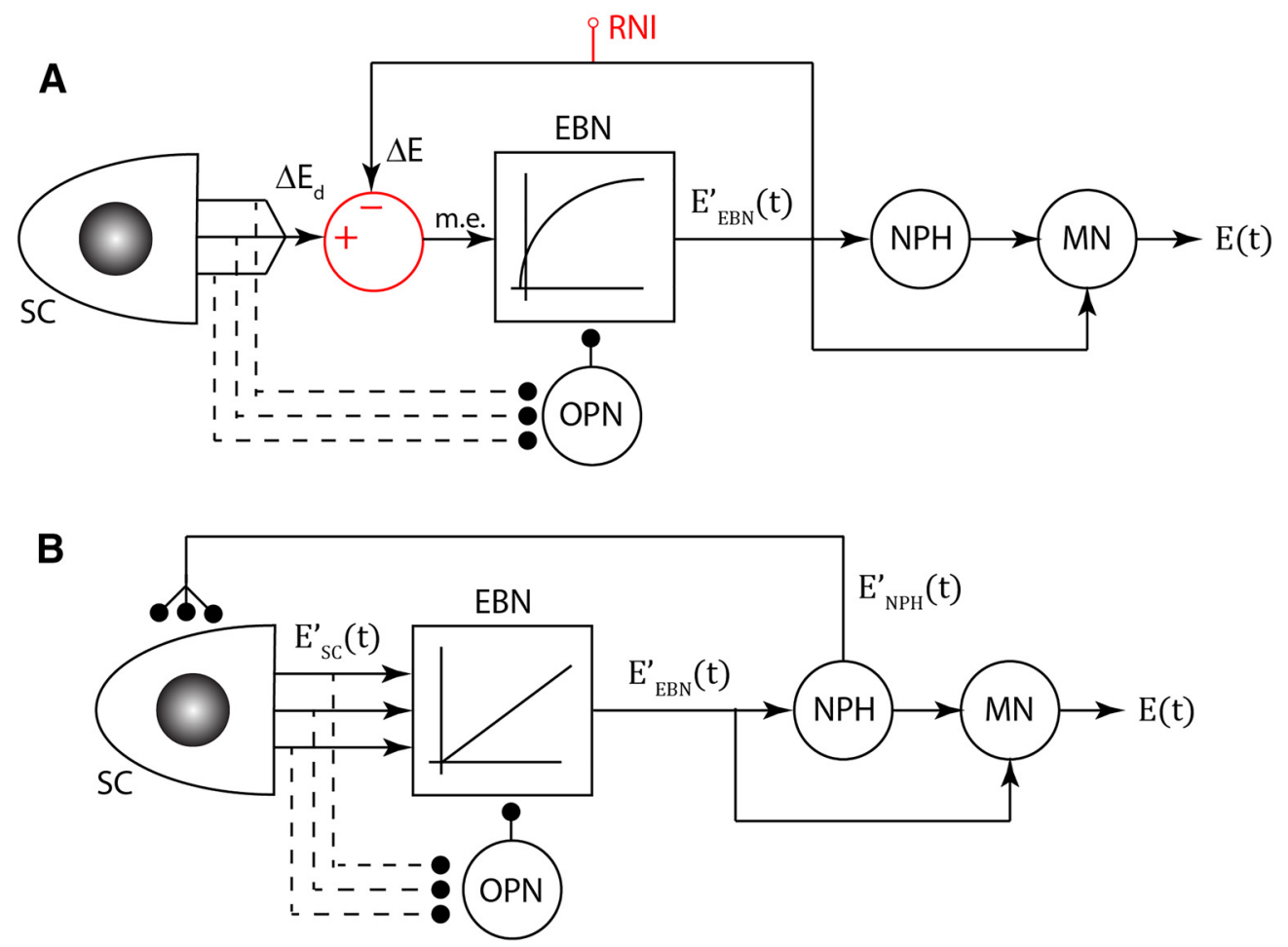

Figure 9. Alternate models of neural control of saccades. $A$, One version of the traditional local feedback loop model. Key elements or processes include computing a desired displacement command $\left(\Delta E_{d}\right)$ from $S$ C population activity, using a comparator (red summation symbol) to compute dynamic motor error (m.e.), and using a resettable neural integrator (RNI; red text) to convert the eye velocity signal $\left(E_{E B N}^{\prime}(t)\right)$ to current eye displacement $(\Delta E)$. Also, the EBN performs a nonlinear transform on motor error signal to determine eye velocity. $B$, Our revised conceptual model is void of the comparator and resettable neural integrator elements and therefore no longer computes dynamic motor error. The EBN output is a linear transform of the $S C$ input. The eye velocity feedback signal $\left(E_{E B N}^{\prime}(t)\right)$ projects to the SC. The spatiotemporal pattern of population SC activity is pivotal in determining the instantaneous eye velocity. MN, Motoneuron.

Downstream of the SC, there are also some salient differences from the traditional framework. Most notably, this scheme does not include the elusive resettable neural integrator and comparator, and the EBN therefore does not operate on motor error. Rather, it only scales the SC signal, where the gain (slope) may be a function of the location of active neurons in the SC; this notion of a linear operation by the EBN is credited to previous work (Goossens and Van Opstal, 2006; van Opstal and Goossens, 2008). Also, the feedback signal now projects to, and is distributed across, the SC, and it originates from the NPH $\left(E_{N P H}^{\prime}(t)\right)$ (Corvisier and Hardy, 1997) because EBNs do not project to the SC. The majority of NPH neurons exhibit burst-tonic patterns, where the burst component resembles eye velocity (Hardy and Corvisier, 1996). Once the movement is initiated, the velocity feedback signal could attenuate the SC burst and contribute to mediating saccade duration. Finally, the engagement of EBN by SC activity may involve nonlinearities other than the OPN gate (not depicted in the figure). For example, the neural trajectory defined by SC population activity may need to traverse in an optimal or potent state space (Churchland et al., 2006b; Kaufman et al., 2014) or must exhibit stable temporal structure (Jagadisan and Gandhi, 2018) to recruit the EBN, but once it is online, it obeys the linear transformation.

The legitimacy of our conceptual model will be determined by its ability to account for saccade properties observed under various experimental conditions. Although we have not yet simulated the model, our intuition can provide some qualitative predictions. Let's first consider the effect of SC stimulation. Guided by previous studies that recorded neural activity simultaneously during microstimulation of the same region (Histed et al., 2009; Logothetis et al., 2010; Vokoun et al., 2010), we do not expect the SC output to mimic the stimulation train, as it appears to do in the pons (Cohen and Komatsuzaki, 1972; Gandhi et al., 2008). Instead, we think the intrinsic connectivity in SC will steer the initial, stimulation-evoked response into an attractor state (Gaussian-like profile) that is similar to the population response associated with an internally generated saccade, thus producing saccades with similar kinematics (Katnani and Gandhi, 2012). We believe the same reasoning applies for chemical inactivation. If the region compromised by the lesion overlaps with the neural tissue activated for a saccade, the intrinsic connectivity will gradually shift the locus, width, and/or firing rate of the spatiotemporal Gaussian pattern (Badler and Keller, 2002), leading to a slower saccade with a potentially different endpoint (Lee et al., 1988). It is also interesting to think about perturbations that alter the trajectory of a saccade or even halt it in midflight. In all cases, the ongoing activity in $\mathrm{SC}$ is perturbed through feedback from the $\mathrm{NPH}$, as shown in Figure $9 B$, or various other methods that are not illustrated (e.g., lateral interactions within the SC, antidromic activation from a site that is stimulated, or through the cerebellum or cortex). The SC population activity, in turn, is reshaped according to the spatiotemporal and refractory dynamics of the network, and the associated eye velocity reflects the SC activity provided that OPN has released its inhibition on the saccade circuitry.

In conclusion, here we present compelling evidence that the SC has dynamic influence over each instant of the saccade. The result impacts the notion of spatiotemporal transformation, which is thought to be a serial process of first encoding the movement in a retinotopic reference frame (place code) and then transforming it into a rate code to control its dynamics (Groh, 2001). The SC is considered the last stage of the spatial represen- 
tation, and gaze centers in the lower brainstem use the temporal algorithm. Our analyses show a clear role of the SC in also exerting temporal control over the saccade throughout the duration of the movement, casting a shadow on a simplistic and serial sensorimotor transformation framework. In doing so, the result aligns well with observations from skeletomotor research, where it was demonstrated decades ago that neurons in the cortex encode velocity of hand movement (Ashe and Georgopoulos, 1994; Reina et al., 2001; Takei et al., 2018).

\section{References}

Ashe J, Georgopoulos AP (1994) Movement parameters and neural activity in motor cortex and area 5. Cereb Cortex 4:590-600. CrossRef Medline

Badler JB, Keller EL (2002) Decoding of a motor command vector from distributed activity in superior colliculus. Biol Cybern 86:179-189. CrossRef Medline

Basso MA, May PJ (2017) Circuits for action and cognition: a view from the superior colliculus. Annu Rev Vis Sci 3:197-226. CrossRef Medline

Bryant CL, Gandhi NJ (2005) Real-time data acquisition and control system for the measurement of motor and neural data. J Neurosci Methods 142: 193-200. CrossRef Medline

Carmena JM, Lebedev MA, Henriquez CS, Nicolelis MA (2005) Stable ensemble performance with single-neuron variability during reaching movements in primates. J Neurosci 25:10712-10716. CrossRef Medline

Churchland MM (2015) Using the precision of the primate to study the origins of movement variability. Neuroscience 296:92-100. CrossRef Medline

Churchland MM, Santhanam G, Shenoy KV (2006a) Preparatory activity in premotor and motor cortex reflects the speed of the upcoming reach. J Neurophysiol 96:3130-3146. CrossRef Medline

Churchland MM, Yu BM, Ryu SI, Santhanam G, Shenoy KV (2006b) Neural variability in premotor cortex provides a signature of motor preparation. J Neurosci 26:3697-3712. CrossRef Medline

Cohen B, Komatsuzaki A (1972) Eye movements induced by stimulation of the pontine reticular formation: evidence for integration in oculomotor pathways. Exp Neurol 36:101-117. CrossRef Medline

Corvisier J, Hardy O (1997) Topographical characteristics of preposito-collicular projections in the cat as revealed by phaseolus vulgaris-leucoagglutinin technique: a possible organisation underlying temporal-to-spatial transformations. Exp Brain Res 114:461-471. CrossRef Medline

Diesmann M, Gewaltig MO, Aertsen A (1999) Stable propagation of synchronous spiking in cortical neural networks. Nature 402:529-533. CrossRef Medline

Edelman JA, Goldberg ME (2001) Dependence of saccade-related activity in the primate superior colliculus on visual target presence. J Neurophysiol 86:676-691. CrossRef Medline

Gandhi NJ, Bonadonna DK (2005) Temporal interactions of air-puffevoked blinks and saccadic eye movements: insights into motor preparation. J Neurophysiol 93:1718-1729. CrossRef Medline

Gandhi NJ, Katnani HA (2011) Motor functions of the superior colliculus. Annu Rev Neurosci 34:205-231. CrossRef Medline

Gandhi NJ, Keller EL (1999) Comparison of saccades perturbed by stimulation of the rostral superior colliculus, the caudal superior colliculus, and the omnipause neuron region. J Neurophysiol 82:3236-3253. CrossRef Medline

Gandhi NJ, Barton EJ, Sparks DL (2008) Coordination of eye and head components of movements evoked by stimulation of the paramedian pontine reticular formation. Exp Brain Res 189:35-47. CrossRef Medline

Goossens HH, Van Opstal AJ (2000) Blink-perturbed saccades in monkey: I. Behavioral analysis. J Neurophysiol 83:3411-3429. CrossRef Medline

Goossens HH, Van Opstal AJ (2006) Dynamic ensemble coding of saccades in the monkey superior colliculus. J Neurophysiol 95:2326-2341. CrossRef Medline

Goossens HH, van Opstal AJ (2012) Optimal control of saccades by spatialtemporal activity patterns in the monkey superior colliculus. PLoS Comput Biol 8:e1002508. CrossRef Medline

Groh JM (2001) Converting neural signals from place codes to rate codes. Biol Cybern 85:159-165. CrossRef Medline

Hardy O, Corvisier J (1996) Firing properties of preposito-collicular neurones related to horizontal eye movements in the alert cat. Exp Brain Res 110:413-424. Medline
Histed MH, Bonin V, Reid RC (2009) Direct activation of sparse, distributed populations of cortical neurons by electrical microstimulation. Neuron 63:508-522. CrossRef Medline

Huang X, Lisberger SG (2009) Noise correlations in cortical area MT and their potential impact on trial-by-trial variation in the direction and speed of smooth-pursuit eye movements. J Neurophysiol 101:3012-3030. CrossRef Medline

Jagadisan UK, Gandhi NJ (2016) Disruption of fixation reveals latent sensorimotor processes in the superior colliculus. J Neurosci 36:6129-6140. CrossRef Medline

Jagadisan UK, Gandhi NJ (2017) Removal of inhibition uncovers latent movement potential during preparation. Elife 6:e29648. CrossRef Medline

Jagadisan UK, Gandhi NJ (2018) Population temporal structure supplements the rate code during sensorimotor transformations. Advance online publication. Retrieved June 11, 2018. doi: 10.1101/132514.

Jürgens R, Becker W, Kornhuber HH (1981) Natural and drug-induced variations of velocity and duration of human saccadic eye movements: evidence for a control of the neural pulse generator by local feedback. Biol Cybern 39:87-96. CrossRef Medline

Kaneda K, Phongphanphanee P, Katoh T, Isa K, Yanagawa Y, Obata K, Isa T (2008) Regulation of burst activity through presynaptic and postsynaptic GABA(B) receptors in mouse superior colliculus. J Neurosci 28:816-827. CrossRef Medline

Kasap B, van Opstal AJ (2017) A spiking neural network model of the midbrain superior colliculus that generates saccadic motor commands. Biol Cybern 111:249-268. CrossRef Medline

Katnani HA, Gandhi NJ (2012) The relative impact of microstimulation parameters on movement generation. J Neurophysiol 108:528-538. CrossRef Medline

Katnani HA, Gandhi NJ (2013) Time course of motor preparation during visual search with flexible stimulus-response association. J Neurosci 33: 10057-10065. CrossRef Medline

Kaufman MT, Churchland MM, Ryu SI, Shenoy KV (2014) Cortical activity in the null space: permitting preparation without movement. Nat Neurosci 17:440-448. CrossRef Medline

Keller EL (1974) Participation of medial pontine reticular formation in eye movement generation in monkey. J Neurophysiol 37:316-332. CrossRef Medline

Keller EL, Edelman JA (1994) Use of interrupted saccade paradigm to study spatial and temporal dynamics of saccadic burst cells in superior colliculus in monkey. J Neurophysiol 72:2754-2770. CrossRef Medline

Kustov AA, Robinson DL (1995) Modified saccades evoked by stimulation of the macaque superior colliculus account for properties of the resettable integrator. J Neurophysiol 73:1724-1728. CrossRef Medline

Lee C, Rohrer WH, Sparks DL (1988) Population coding of saccadic eye movements by neurons in the superior colliculus. Nature 332:357-360. CrossRef Medline

Logothetis NK, Augath M, Murayama Y, Rauch A, Sultan F, Goense J, Oeltermann A, Merkle H (2010) The effects of electrical microstimulation on cortical signal propagation. Nat Neurosci 13:1283-1291. CrossRef Medline

May PJ (2006) The mammalian superior colliculus: laminar structure and connections. Prog Brain Res 151:321-378. CrossRef Medline

Miyashita N, Hikosaka O (1996) Minimal synaptic delay in the saccadic output pathway of the superior colliculus studied in awake monkey. Exp Brain Res 112:187-196. Medline

Morén J, Shibata T, Doya K (2013) The mechanism of saccade motor pattern generation investigated by a large-scale spiking neuron model of the superior colliculus. PLoS One 8:e57134. CrossRef Medline

Munoz DP, Waitzman DM, Wurtz RH (1996) Activity of neurons in monkey superior colliculus during interrupted saccades. J Neurophysiol 75: 2562-2580. CrossRef Medline

Nichols MJ, Sparks DL (1995) Nonstationary properties of the saccadic system: new constraints on models of saccadic control. J Neurophysiol 73: 431-435. CrossRef Medline

Nichols MJ, Sparks DL (1996) Component stretching during oblique stimulation-evoked saccades: the role of the superior colliculus. J Neurophysiol 76:582-600. CrossRef Medline

Osborne LC, Lisberger SG, Bialek W (2005) A sensory source for motor variation. Nature 437:412-416. CrossRef Medline

Phongphanphanee P, Marino RA, Kaneda K, Yanagawa Y, Munoz DP, Isa T 
(2014) Distinct local circuit properties of the superficial and intermediate layers of the rodent superior colliculus. Eur J Neurosci 40:2329-2343. CrossRef Medline

Quaia C, Lefèvre P, Optican LM (1999) Model of the control of saccades by superior colliculus and cerebellum. J Neurophysiol 82:999-1018. CrossRef Medline

Reina GA, Moran DW, Schwartz AB (2001) On the relationship between joint angular velocity and motor cortical discharge during reaching. J Neurophysiol 85:2576-2589. CrossRef Medline

Rezvani S, Corneil BD (2008) Recruitment of a head-turning synergy by low-frequency activity in the primate superior colliculus. J Neurophysiol 100:397-411. CrossRef Medline

Robinson DA (1975) Oculomotor control signals. In: Basic mechanism of ocular motility and their clinical implications (Lennerstrand G, Bach-yRita P, eds), pp 337-374. Oxford: Pergamon.

Rodgers CK, Munoz DP, Scott SH, Paré M (2006) Discharge properties of monkey tectoreticular neurons. J Neurophysiol 95:3502-3511. CrossRef Medline

Schlag J, Pouget A, Sadeghpour S, Schlag-Rey M (1998) Interactions between natural and electrically evoked saccades: III. Is the nonstationarity the result of an integrator not instantaneously reset? J Neurophysiol 79: 903-910. CrossRef Medline

Shmiel T, Drori R, Shmiel O, Ben-Shaul Y, Nadasdy Z, Shemesh M, Teicher M, Abeles M (2006) Temporally precise cortical firing patterns are associated with distinct action segments. J Neurophysiol 96:2645-2652. CrossRef Medline

Sparks DL, Mays LE (1990) Signal transformations required for the generation of saccadic eye movements. Annu Rev Neurosci 13:309-336. CrossRef Medline

Stanford TR, Freedman EG, Sparks DL (1996) Site and parameters of microstimulation: evidence for independent effects on the properties of sac- cades evoked from the primate superior colliculus. J Neurophysiol 76: 3360-3381. CrossRef Medline

Stuphorn V, Hoffmann KP, Miller LE (1999) Correlation of primate superior colliculus and reticular formation discharge with proximal limb muscle activity. J Neurophysiol 81:1978-1982. CrossRef Medline

Takei T, Crevecoeur F, Herter TM, Cross KP, Scott SH (2018) Correlations between primary motor cortex activity with recent past and future limb motion during unperturbed reaching. J Neurosci 38:7787-7799. CrossRef Medline

van Beers RJ (2007) The sources of variability in saccadic eye movements. J Neurosci 27:8757-8770. CrossRef Medline

van Beers RJ (2008) Saccadic eye movements minimize the consequences of motor noise. PLoS One 3:e2070. CrossRef Medline

Van Gisbergen JA, Robinson DA, Gielen S (1981) A quantitative analysis of generation of saccadic eye movements by burst neurons. J Neurophysiol 45:417-442. CrossRef Medline

van Opstal AJ, Goossens HH (2008) Linear ensemble-coding in midbrain superior colliculus specifies the saccade kinematics. Biol Cybern 98:561577. CrossRef Medline

Vokoun CR, Jackson MB, Basso MA (2010) Intralaminar and interlaminar activity within the rodent superior colliculus visualized with voltage imaging. J Neurosci 30:10667-10682. CrossRef Medline

Waitzman DM, Ma TP, Optican LM, Wurtz RH (1991) Superior colliculus neurons mediate the dynamic characteristics of saccades. J Neurophysiol 66:1716-1737. CrossRef Medline

Walton MM, Bechara B, Gandhi NJ (2007) Role of the primate superior colliculus in the control of head movements. J Neurophysiol 98:20222037. CrossRef Medline

Yoshida K, Iwamoto Y, Chimoto S, Shimazu H (2001) Disynaptic inhibition of omnipause neurons following electrical stimulation of the superior colliculus in alert cats. J Neurophysiol 85:2639-2642. CrossRef Medline 